\title{
Combined Scheme for Fast PN Code Acquisition
}

\author{
Salah Sayed Elagooz
}

\begin{abstract}
In direct-sequence wideband (DS-WB) systems, long spreading sequences are used to span multiple symbol intervals in order to remove spectral lines and to mitigate multiple access interference. The usage of long spreading sequences will result in a large search space during acquisition stage. As a result, the DS-WB systems will need a lot of time to achieve acquisition state. This paper proposes a combined acquisition scheme, known as Fast Serial Search Sequential Estimation (FSSSE), which could be applied to the direct sequence spread spectrum systems with long PN sequences. The proposed scheme is a combination of two acquisition schemes which are the serial search acquisition scheme and the rapid acquisition by sequential estimation (RASE) scheme. The proposed scheme gets the advantages of both schemes while overcome their drawbacks. The mean acquisition time of the proposed scheme is reduced 100 times compared to the conventional serial search scheme for PN sequence with a period of $2^{15}-1$.
\end{abstract}

Keywords: Direct-sequence wideband spread spectrum system - PN code acquisition - Serial search acquisition scheme - Fast Serial search sequential estimation acquisition scheme - Rapid acquisition by sequential estimation scheme.

\section{I- Introduction:}

In Direct-Sequence Spread Spectrum (DSSS) systems, the goal of code acquisition is to achieve a coarse time alignment between the received PN code and the locally generated code to an accuracy of a fraction of one PN sequence chip [1, 2].

Serial search strategy [3] is the most popular approach to code acquisition which correlates the received and locally generated code sequences and then tests the synchronization based on either the crossing of threshold or the maximum correlation. A threshold value is determined depending on the signal to noise ratio at the matched filter output [2] and it may be adjusted to the partial correlation [1]. A direct approach [4] for obtaining statistics of the code acquisition time for serial search spread-spectrum receivers is presented. It combines algebraic characterization of the search with transform-domain methods. This approach gives a very deep insight into the nature of the acquisition process. This fact permits the author to propose two alternate search strategies that outperform the conventional ones when the code rewinding time is small in comparison to the dwell times. The serial search scheme has a drawback of its relatively long acquisition time for long periods PN codes. A double-dwell serial code acquisition system employing an adaptive threshold estimator is described and analyzed and a general expression for the mean acquisition time is derived [5].

\footnotetext{
* Egyptian Armed Forces
} 
The performance of a noncoherent [6], serial acquisition technique is evaluated for DSSS communications. The output of a matched filter is sampled at twice the chip rate during acquisition with the advantages of fast acquisition and less hardware. acquisition, and each sample is compared with a fixed acquisition threshold. The effect of sample-timing error on the acquisition performance is examined. A new scheme of PN code acquisition using FIR adaptive filter is presented and it provides better performance than earlier designs of the matched filter [7]. An acquisition scheme [8], named larger step and serial acquisition, of a PN code for DSSS is introduced with a focus on the acquisition problem of long sequences buried deeply in noise. This method is similar to the hybrid

Another approach for PN code acquisition is the recursion-aided rapid acquisition by a sequential estimation (RARASE) [9]. This method is an outgrowth of the (RASE) method, rapid acquisition by sequential estimation. In RARASE method, the known recursion relation of the PN signal is used to determine if a short estimate of the state of the received PN signal is probably correct and if, consequently, an attempt should be made to track that estimate. The RARASE has the drawback of being highly sensitive to noise and interference signals because the estimation process is performed on a chip by chip basis and as such makes no use of the interference rejection capabilities of PN signals. However, it has the advantages of simplicity and fast code acquisition.

For long spreading sequences, as the case of direct-sequence ultra-wideband (DS-UWB) systems [10], an improved signaling format in which the final long spreading sequence used is derived from the combination of two short sequences. According to the feature of the proposed signaling format, a two-stage acquisition scheme is proposed and studied in detail. Results show that the acquisition time can be reduced greatly with the proposed scheme.

This paper presents a new acquisition scheme to account with long spreading sequences that are used in DS-WB systems. This scheme is a hybrid system that combined from a serial search scheme and rapid acquisition by sequential estimation scheme. This system can be expressed as: fast serial search sequential estimation (FSSSE) scheme, which carries the state information of long period PN code generator over another short period PN sequence. This scheme gets the advantages of both serial search and sequential estimation schemes while overcomes their drawbacks.

The paper is organized as follows: Section II provides the basic principle of the FSSSE acquisition scheme. Section III describes the implementation and operation of the acquisition scheme. Section IV drives its performance. Section V shows the results. Section VI gives the conclusion.

\section{II- Basic Principle of the FSSSE Acquisition Scheme}

One can synchronize two linear feedback shift register generators of the same structure by loading the same initial states in the two shift registers at nearly the same time. So, if we can transmit this initial state and estimate its values at the receiver side, the system can acquire synchronization in a fast manner. In order to do that, one should solve the problem of how to transmit the initial state of the code generator in practical low signal to noise ratio and then, how to correctly estimate this initial state at the receiving side. The proposed FSSSE system can solve these problems. 
Acquisition strategy in FSSSE system is achieved in three steps. First, a short period PN sequence, known as synchronization sequence, is synchronized in a very short time due to its short period. This synchronization sequence is used as a carrier for the initial state of the main long period PN sequence. Second, the initial state of the main sequence is estimated out of the synchronization sequence. Third, the long period PN code sequence is finally synchronized by applying the estimated initial state to the long period shift register generator at the receiving side. The operation of each of the three steps takes a relatively very short time in a low SNR environment so that, the overall acquisition time becomes very short comparing with either of the two individually working schemes.

\section{III- Implementation and Operation of FSSSE Acquisition Scheme}

The data to be transmitted is spreaded by a main long period PN code sequence. The spreading long period PN sequence it self is spreaded by another fast short period PN sequence, which can be considered as a carrier signal for the long PN code sequence. The basic building blocks for the transmitter module are shown in Fig.1

At the receiving side shown in Fig. 2, the locally generated short period PN sequence is firstly synchronized using a serial search acquisition scheme because it is more practical in a low SNR environment. When the system achieves synchronization for the short PN sequence, the state signal is estimated and loaded into the long code generator to perform synchronization of the long PN sequence using the RASE scheme. As the two steps take very short times then, the overall system acquisition is achieved in a relatively short time.

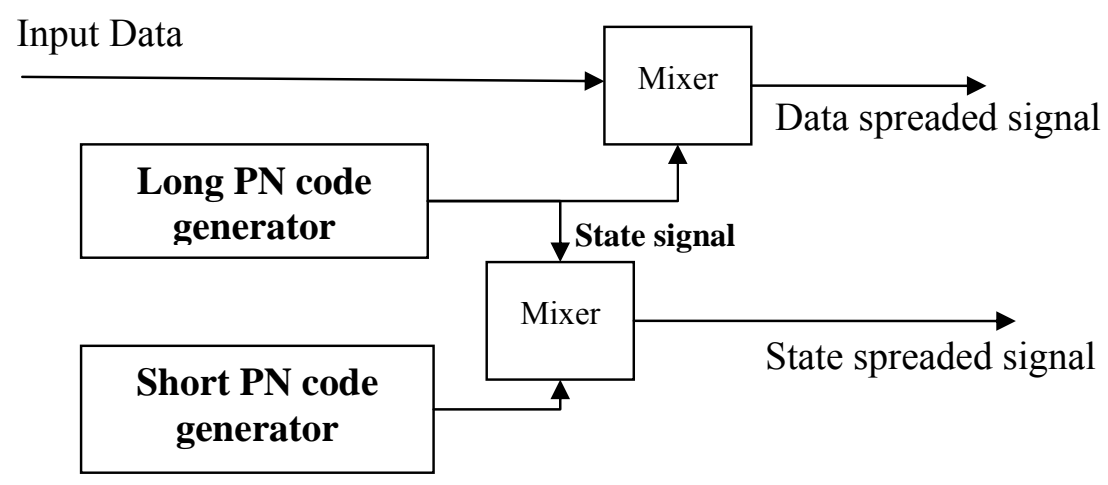

Fig. 1 Transmitter block diagram. 
Received short period sequence (State Signal)
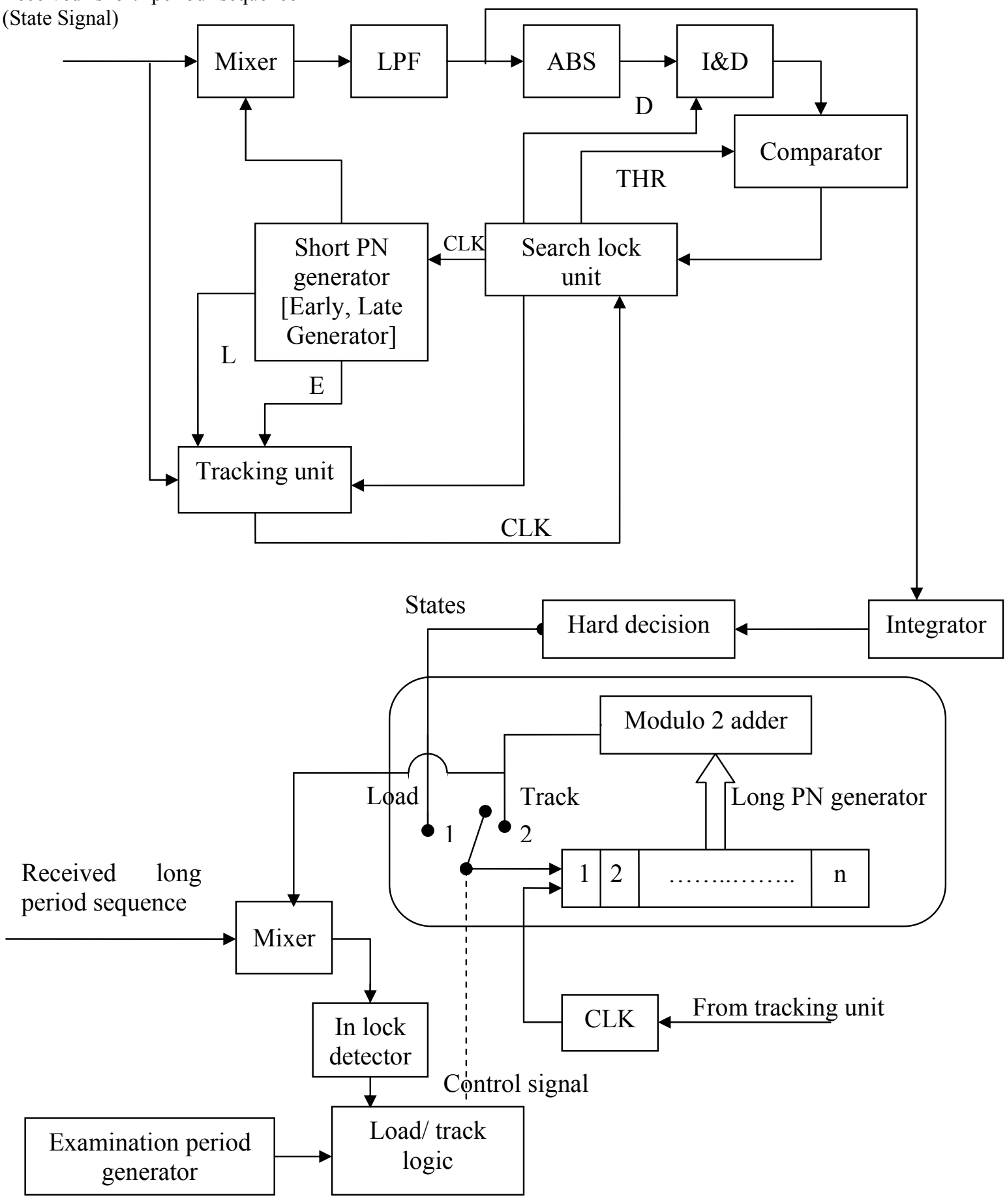

Fig. 2 Receiver Block Diagram. 


\section{IV- Performance of FSSSE Acquisition Scheme}

Because the FSSSE scheme is a combination of double dwell acquisition scheme (serial search strategy) [3] and RASE scheme [9] so, the average acquisition time of the all system is obtained by averaging the acquisition times of the two schemes.

Double dwell acquisition scheme is one of the fastest serial search strategies; it depends on using two different dwell (integration) times. The first one is the shortest time and therefore it results in a high probability of detection $\mathrm{P}_{\mathrm{D}}$ and a high probability of false alarm $\mathrm{P}_{\mathrm{FA}}$. So, this allows neglecting unsynchronized cells rapidly. The second dwell time is longer with low $P_{D}$ and low $\mathrm{P}_{\mathrm{FA}}$, these results in a reduction in the overall acquisition time.

It can be shown that the performance in terms of the average acquisition time $\bar{T}_{\text {acq }}$ and the acquisition time variance $\sigma_{\mathrm{acq}}{ }^{2}$ for the double dwell acquisition scheme is given by $[1,4]$ :

$$
\begin{aligned}
\bar{T}_{a c q} & =\frac{\left(2-P_{L}\right) q \bar{\tau}_{d}}{2 P_{L}}, \\
\sigma_{a c q}^{2} & =\bar{\tau}_{d}^{2} q^{2}\left(\frac{1}{12}+\frac{1}{P_{L}^{2}}-\frac{1}{P_{L}}\right),
\end{aligned}
$$

where $\mathrm{P}_{\mathrm{L}}=\mathrm{P}_{\mathrm{D} 1} \mathrm{P}_{\mathrm{D} 2}$, q is the number of cells to be tested $\mathrm{q}=2 \mathrm{~N}, \mathrm{~N}$ is the PN code length $\mathrm{N}=$ $2^{\mathrm{n}}-1, \mathrm{n}$ is the length of the shift register, and $\mathrm{P}_{\mathrm{D} 1}, \mathrm{P}_{\mathrm{D} 2}$ are the probabilities of detection for the first and the second dwell times respectively. The average dwell time $\bar{\tau}_{d}$ is given by:

$$
\bar{\tau}_{d}=\tau_{d 1}+\tau_{d 2} P_{F A 1}+T_{p} P_{F A 1} P_{F A 2} \text {, }
$$

where $\tau_{\mathrm{d} 1}$ is the first dwell time, $\tau_{\mathrm{d} 2}$ is the second dwell time of the scheme, and $\mathrm{P}_{\mathrm{FA} 1}, \mathrm{P}_{\mathrm{FA} 2}$ are the probabilities of false alarm for the first and second dwell times respectively. The total penalty time is given by:

$\mathrm{T}_{\mathrm{p}}=\mathrm{K} \tau_{\mathrm{d} 2}$

where $\mathrm{K}$ is the number of penalty times.

The mean acquisition time for RASE scheme is evaluated as follow [9]. Let p denotes the probability of correctly estimating a particular received symbol; this probability is a function of received SNR. The probability of correctly loading the shift register of length $n$ in a single trial is $\mathrm{p}^{\mathrm{n}}$, and the probability of an incorrect shift register load is $1-\mathrm{p}^{\mathrm{n}}$. The probability of obtaining a correct load on the $\mathrm{k}^{\text {th }}$ trial is given by:

$$
p_{r}(k)=p^{n}\left(1-p^{n}\right)^{k-1}
$$

The average number of trials required to achieve a correct load is:

$$
\begin{aligned}
& \bar{k}=\sum_{k=1}^{\infty} k p_{\mathbf{r}}(\mathbf{k}), \\
& \bar{k}=\sum_{\mathbf{k}=1}^{\infty} \mathbf{k p}^{\mathrm{n}}\left(1-\mathbf{p}^{\mathrm{n}}\right)^{\mathrm{k}-1},
\end{aligned}
$$


Using the substitution $\mathrm{q}=1-\mathrm{P}^{\mathrm{n}}$ and $\mathrm{m}=\mathrm{k}-\mathrm{l}$ along with the identity

$\sum(a+m) q^{m}=\frac{a}{(1-q)}+\frac{q}{(1-q)^{2}}$,

Finally, the average number of trials required to achieve a correct load is:

$$
\bar{k}=\frac{1}{p^{n}} .
$$

Each shift register requires $\mathrm{nT}_{\mathrm{c}}$ seconds plus $T_{\mathrm{e}}$ (verification time) seconds to evaluate the correctness of the load. Ignoring the possibility that a correct load could be misidentified as an incorrect load, the time required for each trial is $\left(n T_{c}+T_{e}\right)$ and the mean acquisition time for this system will be:

$$
\overline{T_{s}}=\bar{k}\left(n T_{c}+T_{e}\right)=\frac{n T_{c}+T_{e}}{p^{n}},
$$

Where $T_{c}$ is the chip period and $T_{e}$ is the verification time or the examination period.

Finally, the over all average acquisition time of the FSSSE scheme is the summation of the average acquisition times of the double dwell acquisition scheme and the RASE acquisition scheme.

Tacq $=\left[(2-\mathrm{PL}) \mathrm{q}^{\overline{\tau_{d}}} / 2 \mathrm{PL}\right]+[(\mathrm{nTc}+\mathrm{Te}) / \mathrm{Pn}]$.

One should notice that in FSSSE acquisition scheme, the first step of acquisition, using the double dwell acquisition scheme, is done using a short period PN sequence (initial state carrier signal) instead of the long period sequence and hence, the average acquisition time is highly reduced specially in a low SNR environment.

\section{V- Results:}

The performance of the FSSSE acquisition scheme is obtained in terms of average time to synchronize as a function of SNR, taking into consideration the presence of AWGN. The obtained result is compared separately with each of the double dwell serial search acquisition scheme and the RASE acquisition scheme for the same code length.

Fig.3 provides the average acquisition time of the double dwell acquisition scheme, the RASE acquisition scheme, and the FSSSE scheme as a function of SNR. From the figure one can notice the following:

1- The average acquisition time of FSSSE scheme has a comparable average acquisition time with the RASE acquisition scheme at moderate to high SNR.

2- The RASE acquisition scheme has a low acquisition time at moderate to high SNR. However, this system is not working at low values of SNR.

3- The average acquisition time of FSSSE scheme is much less then average acquisition time of the double dwell acquisition scheme that works individually.

4- The FSSSE acquisition scheme provides the lowest average acquisition time when working in a low SNR environment. 




Fig. 3 The average acquisition time of the double dwell scheme, the RASE scheme, and the FSSSE scheme as a function of SNR in [db].

\section{VI- Conclusions:}

A proposed combined acquisition scheme for direct sequence spread spectrum known as fast serial search sequential estimation acquisition scheme has been proposed. This scheme gets the advantages of both serial search acquisition scheme and RASE acquisition scheme, while overcomes their drawbacks.

The FSSSE proposed scheme provides the lowest average acquisition time, especially for long period PN codes under a low signal to noise ratio working conditions, when compared with each of the double dwell serial search acquisition scheme and the RASE acquisition scheme that work individually. 


\section{VII- References}

1- M. K. Simon, J. K. Omura, R. A. Scholtz, and B. K. Levitt, spread spectrum communication handbook. New York: McGraw- Hill, 2000.

2- S. S. Rappaport and D. M. Grieco, "Spread-spectrum signal acquisition Methods and technology.” IEEE Commun. Mag., vol. 22, pp. 6-21, June 1984.

3- A. Polydoros and C. Weber, "A unified approach to serial search spread-spectrum code acquisition,” IEEE Trans Commun. Vol. COM-32, pp. 542-560, May 1984.

4- Jovanovic and Vladan M. "Analysis of strategies for serial-search spread-spectrum code acquisition - Direct approach," Trans. Commun., vol. COM-36, 1208-1220, Nov.1988.

5- Hyung-Rae Park and Bub-Joo Kang, "On serial search code acquisition for directsequence spread spectrum system: an application to IS-95 CDMA system," Vehicular Technology Conference, IEEE 45th Volume 1, Issue , 25-28, pp. 291 - 295, Jul 1995.

6- Noneaker, D.L. , "The performance of serial, matched-filter acquisition in direct-sequence packet radio communications," Military Communications Conference, MILCOM 2001. Vol.2, pp.1045- 1049, 2001.

7- Asoodeh, S.and Maddahi, H., "On PN code acquisition in direct sequence code division multiple access," International Conference on Wireless Communications, Networking and Mobile Computing, WiCom, pp. 1433-1436, Sept. 2007

8- Feng She, Dingjie Xu and Bing Xue "An approach to DS-SS signal acquisition using larger step and serial acquisition algorithm," International Conference on Mechatronics and Automation, ICMA, pp. 3492-3496, Aug. 2007.

9- R. B. Ward and K. P. Yiu, "Acquisition of pseudonoise signals by recursion aided sequential estimation,” IEEE Trans. Commun., vol. COM-25, pp. 784-794. Aug. 1977.

10- Peng Wang and Deqiang Wang "Acquisition scheme for DS ultra-wideband with combined spreading sequence," International conference on wireless communications, networking and mobile computing, WiCom, pp. 508-511, Sept. 2007 